\title{
Achnanthidium exiguum (Bacillariophyta): nuevas citas para localidades andinas del norte de Chile
}

\section{Achnanthidium exiguum (Bacillariophyta): new reports from Andean localities in northern Chile}

\author{
Patricio Rivera* \& Fabiola Cruces \\ Departamento de Botánica, Universidad de Concepción, Casilla 160-C, Concepción, Chile. \\ *privera@udec.cl
}

\begin{abstract}
Achnanthidium exiguum is reported for the first time from Salar de Huasco and Salar de Aguas Calientes in northern Chile. The morphological features of the species are described and illustrated with light and electron microscopy photograhs, and agree well with the type material studied by Schoeman \& Archibal in 1976. The distribution of the species in Chile is also given.
\end{abstract}

El género Achnanthidium Kützing está ampliamente repartido en las aguas dulces de todo el planeta. Pertenece al orden Achnanthales que incluye a los individuos que son heterovalvares debido a la presencia de rafe en una valva y ausencia del mismo en la otra valva, y algunas de sus características son el poseer un tamaño reducido, estrías uniseriadas alrededor de 30 en 10 micrometros y más espaciadas en las valvas sin rafe, extremos proximales del rafe notoriamente expandidos y extremos distales derechos o girados hacia un mismo lado (Round \& Bukhtiyarova, 1996).

Achnanthidium exiguum (Grunow) Czarnecki es una especie cosmopolita muy variable con relación a sus características morfológicas, lo que ha llevado a la descripción de diversas variedades y formas, dos de ellas citadas anteriormente para aguas chilenas: var. heterovalva Krasske 1923 y var. constricta (Torka) Hustedt 1930. Una revisión del material tipo de esta especie (Trinidad y Tobago, Kützing 1844 como Stauroneis exilis, Museo Británico) mediante técnicas modernas de microscopía fotónica y electrónica de transmisión, fue llevada a cabo por Schoeman \& Archibald en 1976. Estas observaciones y otras, realizadas en material recolectado en África del Sur e Indonesia, llevaron a estos investigadores a considerar que las variedades mencionadas anteriormente para Chile son nombres sinónimos de la variedad tipo. Más recientemente un duplicado del material de Kützing 1844 depositado en la Colección de Van Heurck, Bélgica fue reestudiado por, Taylor et al. (2014), empleándose, además de microscopia fotónica, técnicas de microscopía electrónica de barrido. Los autores concluyeron que el material citado como
Achnanthidium exiguum para las Islas Shetland del Sur en el Territorio Chileno Antártico, presenta características morfológicas diferentes al material tipo de $A$. exiguum (contorno valvar, mayor tamaño de los individuos, un bien desarrollado estauro en la valva sin rafe, entre otras), lo que les permitió describir a Achnanthidium australexiguum Van de Vijver sp. nov., un taxón relacionado con $A$. exiguum var. angustirostrata (Krasske) Lange-Bertalot descrito en 1939 para aguas chilenas. Tanto $A$. exiguum como $A$. australexiguum presentan los extremos distales del rafe curvados en distinta dirección, lo que es una excepción al género.

El análisis de muestras depositadas en la Colección Diatomológica de la Universidad de Concepción permitió reconocer la presencia de $A$. exiguum en humedales de dos salares ubicados a gran altura en el norte de Chile, el Salar de Huasco y el Salar de Aguas Calientes, donde no había sido reportado anteriormente. El Salar de Huasco se encuentra en la Primera Región de Tarapacá, Chile (20¹8'S-68 52'W a una altura de $3.778 \mathrm{~m}$ s.n.m. y ocupa una superficie de 51 $\mathrm{km}^{2}$. Las lagunas superficiales son de extensión variable y de poca profundidad. Las aguas son de calidad aceptable para el consumo humano y para riego, siendo el arsénico y el boro los principales contaminantes. El Salar de Aguas Calientes, ubicado a 4.200 m s.n.m. en la Segunda Región de Antofagasta $\left(23^{\circ} 30^{\prime} \mathrm{S}-67^{\circ} 33^{\prime} \mathrm{W}\right)$ tiene una superficie de $134 \mathrm{~km}^{2}$. El aporte de agua desde el Río Pili y desde la Quebrada de Chamaca permite la formación de vegas al desembocar en el salar. Las aguas son en general salobres y saladas (Risacher et al. 1999). 

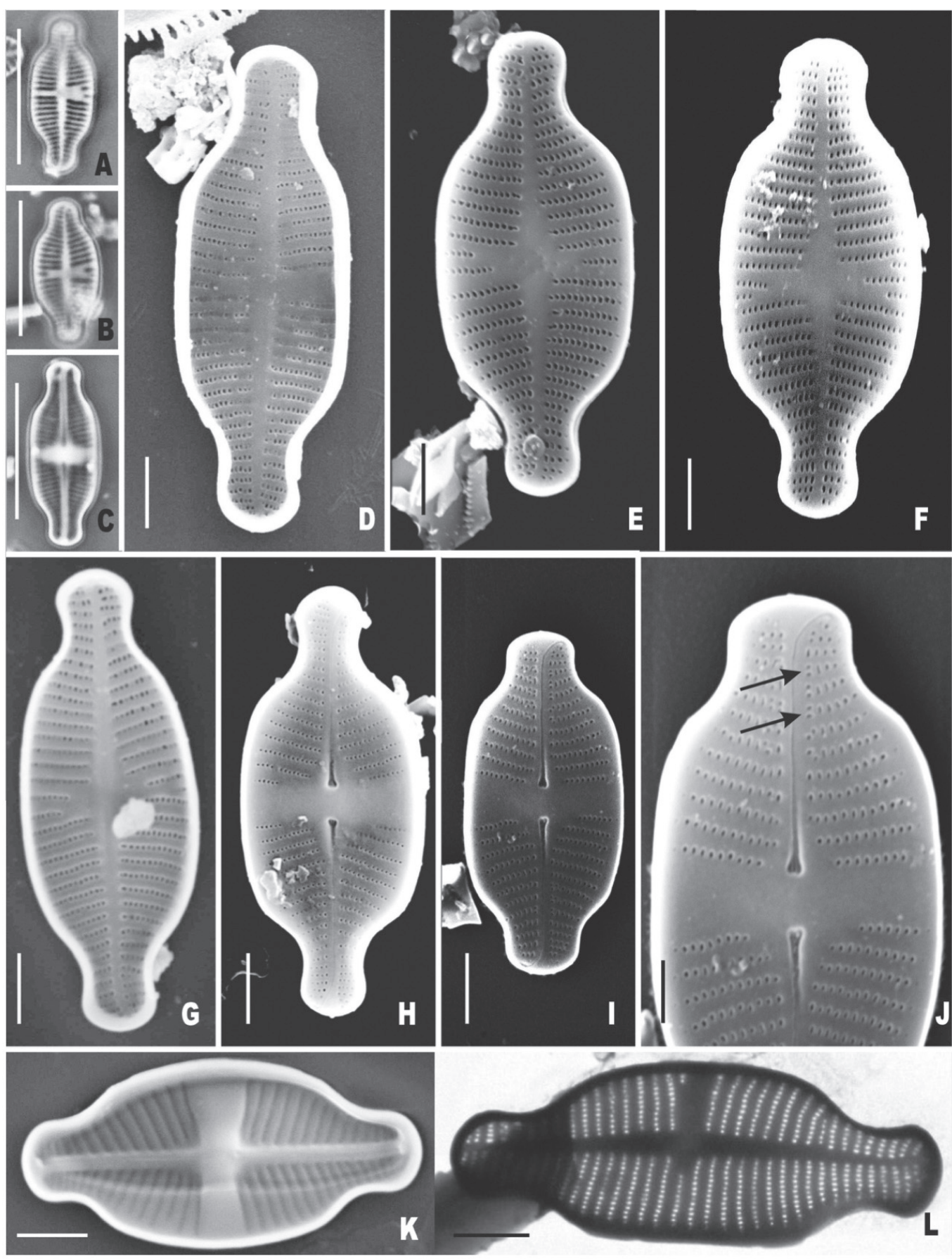

Figura 1. Achnanthidiun exiguum. A-C, Microscopía Fotónica; D-K, Microscopía Electrónica de Barrido: L, Microscopía Electrónica de Transmisión. A-C. Variación del contorno valvar y del área central. D-G. Valvas sin rafe. Area central asimétrica, con fascia ó con una, dos o raramente tres estrías cortas. Extremos subcapitados. H-J. Valvas con rafe, exterior. Area central asimétrica alcanzando el margen valvar, sin estrías. Las flechas indican aréolas biseriadas. Note las fisuras distales del rafe curvadas en direcciones opuestas. K. Estauro en vista interior de valva con rafe. L. Valva sin rafe con un lado convexo y el otro linear; el sternum se ensancha desde los polos hacia el área central. Escalas: A-C $=10 \mu \mathrm{m}$; D-I, K-L $=2 \mu \mathrm{m} ; \mathrm{J}=1 \mu \mathrm{m}$. / Achnanthidium exiguum. A-C, Light Microscopy; D-K, Scanning Electron Microscopy; L, Transmission Electron Microscopy. A-C. Shape of valves and central area variation. D-G. Rapheless valves. Central area asymmetric, with fascia or with one, two, rarely three short striae. Apices subcapitates. H-J. External view of raphe valves. Central area asymetric, reaching the valve margins; shortened striae are not present. Arrows indicate biseriate striae. Note distal rafe fisures deflected to opposite side. K. Interior view of raphe valve with stauro. L. Rapheless-valve with a convex and a more linear margins; sternum widening from the poles to the central area. Scale bars; A-C $=10 \mu \mathrm{m} ; \mathrm{D}-\mathrm{I}, \mathrm{K}-\mathrm{L}=2 \mu \mathrm{m} ; \mathrm{J}=1 \mu \mathrm{m}$. 
Con anterioridad la especie fue señalada en Chile (como Achnanthes exigua Grunow) para el Trípoli de San Pedro de Atacama (Frenguelli 1934), Río Carampangue (Rivera \& Valdebenito 1979), Trípoli de la Isla Cailín, Chiloé (Frenguelli 1935), Isla Dawson; SW de Bahía Inútil; San Isidro, Cabo Froward; Mallin Chileno (Krasske 1949); Loa-Atacama (Hustedt 1927); Río Cauquenes, Chile Central (Rumrich et al. 2000). Como Achnanthes exigua var. constricta (Grunow) Hustedt fue citada para la Bahía de Coquimbo (Rivera 1974), y como Achnanthes exigua var. heterovalva Krasske para Calbuco; Río Puelo; Lago Puyehue; Termas de Puyehue; Lago Risopatrón; tributario del Lago Risopatrón; Río Pascua (Krasske 1939); Tierra del Fuego (Cleve-Euler 1948).

La materia orgánica de los frústulos fue removida de acuerdo con el método de Teubner (1995). Se analizaron cerca de 85 valvas mediante microscopía fotónica (Olympus CX-31), electrónica de barrido (JEOL JSM-6380 LVY) y transmisión (JEOL 1200 EX II). El material estudiado se encuentra depositado en la Colección Diatomológica de la Universidad de Concepción, Chile, DIAT-CONC. La terminología empleada está de acuerdo con Ross et al. (1979) y Round et al. (1990).

Sólo frústulos aislados fueron encontrados. Las valvas son elípticas, con los márgenes casi siempre convexos pero a veces uno (Fig. 1L), o ambos lados (Fig. 1D), son más lineares; los extremos varían de cortamente rostrados (Fig. 1I) a subcapitados, redondeados (Figs. 1C, F-H, L). Eje apical 10.5 - $14.5 \mu \mathrm{m}$; eje transapical 4.7 - $5.6 \mu \mathrm{m}$. Valva sin rafe: el sternum se ensancha gradualmente desde los polos hacia el centro donde forma un área central (Figs. 1DG, L) asimétrica y de forma variable. El área central puede presentar a un lado una fascia que alcanza el borde valvar y al otro lado una estría corta (Fig. 1D), o puede poseer una (Fig. 1B), dos (Fig. 1E) o raramente tres (Fig. 1F) estrías más cortas a cada lado. Las estrías son radiales en el centro de la valva, raramente algo paralelas (Fig. 1L), 20-23 en $10 \mu \mathrm{m}$ y más numerosas en los extremos, 31-36 en $10 \mu \mathrm{m}$. Valva con rafe: rafe sternum recto y rafe filiforme con extremos proximales dilatados y fisuras distales curvadas en direcciones opuestas en vista externa (Figs. 1H-J) y extremos centrales no dilatados y distales terminados en una pequeña helictoglossa en vista interna (Fig. 1K). Área central asimétrica, con estauro (Fig. 1K) ensanchado transversalmente con forma de ala de mariposa que alcanza los márgenes de la valva, carente de estrías. Estrías radiales, 22-25 en $10 \mu \mathrm{m}$ en el centro y 33-40 en $10 \mu \mathrm{m}$ en los extremos.

Mediante microscopía electrónica se observa que las estrías son uniseriadas, con aréolas de contorno levemente alargado (Figs. 1E-F). Sin embargo, cerca de los extremos de algunas valvas con rafe existen unas pocas estrías biseriadas, próximas al rafe sternum (Fig. 1J, flechas). Algunos caracteres descritos por Taylor et al. 2014 en el duplicado del material de Kützing (Bélgica) como valvas con estrías casi paralelas en la parte central de la valva sin rafe, esternum linear no ensanchado hacia el área central y extremos valvares solamente rostrados y no subcapitados, no han sido observados en los especímenes chilenos. Las características de estos especímenes son más cercanas con el material tipo, africano e indonésico, analizado por Schoeman y Archibald (1976).

Achnanthidium exiguum es una especie con una enorme distribución geográfica desarrollándose bajo variadas condiciones ecológicas (Bey \& Ector 2013). Tolera altas temperaturas, teniendo su óptimo desarrollo en aguas alcalinas con moderado a elevado contenido electrolítico (Taylor et al. 2007).

Material Estudiado

CHILE: DIAT-CONC M. 3210. Humedales Salar de Huasco.

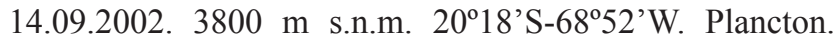
DIAT-CONC M. 3214. Humedales Salar de Huasco. Puerto Batea. 14.09.2002. 3800 m s.n.m. $20^{\circ} 18^{\prime}$ 'S-68 $52^{\prime}$ 'W. DIATCONC M. 3298. Salar de Aguas Calientes. 20.07.2002. 4200 m s.n.m. $23^{\circ} 30^{\prime}$ S $-67^{\circ} 33^{\prime} \mathrm{W}$.

\section{AGRADECIMIENTOS}

Agradecemos a dos revisores anónimos por sus sugerencias y comentarios al manuscrito. Reconocemos también la ayuda otorgada por el personal del Centro de Espectroscopía y Microscopía Electrónica de la Universidad de Concepción. Proyecto parcialmente subsidiado por la Dirección de Investigación, Universidad de Concepción.

\section{REFERENCIAS}

Bey, M., Ector, L. 2013. Atlas des diatomées des cours d'eau de la región Rhone-Alpes. Direction Régionale de l'Environnment, de l'Aménagement et du Longement Rhone-Alpes. Tome 1, pp. 96-97. Bonn'Impression 69300 Caluire.

Cleve-Euler, A. 1948. Süsswasserdiatomeen aus dem Feuerland. Acta Geographica (Scietas Geographica Fennicae) 10(1): $1-61$.

Frenguelli, J. 1934. Diatomeas del Trípoli de San Pedro de Atacama. Revista Chilena de Historia Natural 38: 159-163.

Frenguelli, J. 1935. Análisis diatomológico de trípolis chilenos. Revista Chilena de Historia Natural 39: 147-152.

Hustedt, F. 1927. Fossile Bacillariaceen aus dem Loa-Becken in der Atacama-Wüste, Chile. Archiv für Hydrobiologie und Planktonkunde 18: 224-251.

Hustedt, F. 1930. Bacillariophyta (Diatomeaea). In Pascher A. (ed.): Die Süsswasser-Flora Mitteleuropas. Zweite Auflage, Heft 10, 466 S., Gustav Fisher, Jena.

Krasske, G. 1923. Die Diatomeen des Casseler Beckens und. seiner Randgebirge, nebst einigen wichtigen funden aus 
Niederhessen. Botanisches Archiv 3(4): 185-209.

Krasske, G. 1939. Zur Kiesealgenflora Südchiles. Archiv für Hydrobiologie 35: 349-468.

Krasske, G. 1949. Subfossile Diatomenn aus den Mooren patagoniens und Feuerlands. Suomalaisen Tiedeakatemian Toimituksia, Annales Academie Scientiarum Fennicae, Sarja Series A, IV, Biologica, V. 14. 94 S.

KütZING, F.T. 1844. Die Kieselschaligen Bacillarien oder Diatomeen. Norhausen. $152 \mathrm{~S}$.

Risacher, F., Alonso, H., Salazar, C. 1999. Geoquímica de aguas en cuencas cerradas: I, II y III Regiones- Chile. Ministerio de Obras Públicas. Dirección General de Aguas. Vol. 3. pp. II 145- II 154.Universidad Católica del Norte, Institut de Recherche pour le Développement. Convenio de Cooperación DGA.UCN.IRD.

RiverA, P. 1974. Diatomeas epífitas en Gracilaria verrucosa (Hudson) Papenfuss recolectada en la costa chilena. Gayana Botánica 25: 1-115.

Rivera, P., Valdebenito, H. 1979. Diatomeas recolectadas en las desembocaduras de los Ríos Chivilingo, Laraquete y Carampangue, Chile. Gayana Botánica 35: 1-99.

Ross, R., Cox, E.J., Karayeva, N.I., Mann, D.G., Paddock, T.B.B., Simonsen, R., Sims, P.A. 1979. An amended terminology for the siliceous components of the diatom cell. Nova Hedwigia Beihefte. 64: 513-533.
Round, F.E., Bukhtiyarova, L. 1996. Four new genera based on Achnanthes (Achnanthidium) together with re-definition of Achnanthidium. Diatom Research 11: 345-361.

Round, F.E., Crawford, R.M., Mann, D.G. 1990. The diatoms. Biology and morphology of the genera. Cambridge University Press, Cambridge. 747 pp.

Rumrich, U., Lange-Bertalot, H., Rumrich, M. 2000. Diatomeen der Anden. Von Venezuela bis Patagonien- Tierra del Fuego. In: Lange-Bertalot, H. (ed.). Iconographia Diatomologica 9: 1-673.

Schoeman, F.R., Archibald, R.E.M. 1976. The diatom flora of South Africa. Part 3. C.S.I.R. Special Reports, Pretoria.

Taylor, J.C., Harding, W.R., Archibald, C.G.M. 2007. An Illustrate Guide to Some Common Diatom Species from South Africa. WRC Report TT 282/07, South Africa. 11 pp.

Taylor, J.C., Cocquyt, C., Karthick, B., Van de vijver, B. 2014. Analysis of the type of Achnanthes exigua Grunow (Bacillariophyta) with the description of a new Antarctic diatom species. Fottea, Olomouc 114(1): 43-51.

Teubner, K. 1995. A light microscopical investigation and multivariate statistical analyses of heterovalvar cells of Cyclotella-species (Bacillariophyceae) from lakes of the Berlin-Brandeburg region. Diatom Research 10:191-205.

Recibido: 25.01 .2018

Aceptado: 15.05.2018 